\title{
Edge Detection and Ridge Detection with Automatic Scale Selection
}

\author{
Tony Lindeberg \\ Computational Vision and Active Perception Laboratory (CVAP)* \\ KTH (Royal Institute of Technology), Stockholm, Sweden
}

\begin{abstract}
When extracting features from image data, the type of information that can be extracted may be strongly dependent on the scales at which the feature detectors are applied. This article presents a systematic methodology for addressing this problem. A mechanism is presented for automatic selection of scale levels when detecting onedimensional features, such as edges and ridges.

A novel concept of a scale-space edge is introduced, defined as a connected set of points in scale-space at which: (i) the gradient magnitude assumes a local maximum in the gradient direction, and (ii) a normalized measure of the strength of the edge response is locally maximal over scales. An important property of this definition is that it allows the scale levels to vary along the edge.

Two specific measures of edge strength are analysed in detail. It is shown that by expressing these in terms of $\gamma$ normalized derivatives, an immediate consequence of this definition is that fine scales are selected for sharp edges (so as to reduce the shape distortions due to scale-space smoothing), whereas coarse scales are selected for diffuse edges, such that an edge model constitutes a valid abstraction of the intensity profile across the edge.

With slight modifications, this idea can be used for formulating a ridge detector with automatic scale selection, having the characteristic property that the selected scales on a scale-space ridge instead reflect the width of the ridge.
\end{abstract}

\section{Introduction}

One of the most intensively studied subproblems in computer vision concerns how to detect edges from image data. The importance of edge information for early machine vision is usually motivated from the observation that under rather general assumptions about the image formation process, a discontinuity in image brightness can be assumed to correspond to a discontinuity in either depth, surface orientation, reflectance or illumination.

A non-trivial aspect of edge based analysis of image data, however, concerns what should be meant by a discontinuity in image brightness. Real-world image data are inherently discrete, and for a function defined

\footnotetext{
*The support from the Swedish Research Council for Engineering Sciences, TFR, is gratefully acknowledged. Address: KTH, NADA, S-100 44 Stockholm, Sweden Email: tony@bion.kth.se $W W W:$ http://www.bion.kth.se/ 〜ony
}

on a discrete domain, there is no natural notion of a "discontinuity". This means that there is no inherent way to judge what are the edges in a given discrete image. Therefore, the concept of an image edge is only what we define it to be.

Since the pioneering work by Roberts (1965), a large number of approaches have been developed for detecting edges. Early schemes, such as the Sobel operator and the Prewitt operator, focused on the detection of points at which the gradient magnitude was high, and computed derivative approximations either directly from the pixels or using local least-squares fitting (Haralick 1984). Torre and Poggio (1980) as well as Marr and Hildreth (1980) motivated the need for a smoothing operator to precede differentiation, Canny (1986) considered the problem of determining an optimal smoothing filter of finite support constituting the "best" trade-off between detection and localization properties, given a constraint on the probability of obtaining multiple responses to a single edge. Deriche (1987) extended this approach to filters with infinite support. Similar concepts were developed by Korn (1988). Bergholm (1987) proposed to track edges from coarse to fine scales.

Today, one example of a state-of-the-art edge detector consists of pre-smoothing the image by a Gaussian kernel followed by non-maximum suppression. The latter corresponds to detecting points at which the gradient magnitude assumes a maximum in the gradient direction, and can be given either an algorithmic or differential geometric definition. In this way, edges can be detected at any scale in scale-space (Lindeberg 1994).

The subject of this article is to extend the abovementioned ideas to include the scale dimension already in the edge definition, to simultaneously allow for automatic determination of scale levels appropriate for extracting a given edge. To illustrate the need for such a mechanism, fig. 1 shows the result of computing edges from an image at a number of different scales. As can be seen, different types of edge structures give rise to edge curves at different scales. For example, the shadow of the arm only appears as a connected edge curve at coarse scales. If such coarse scales are used at the finger tip, however, the shape distortions due to scale-space 

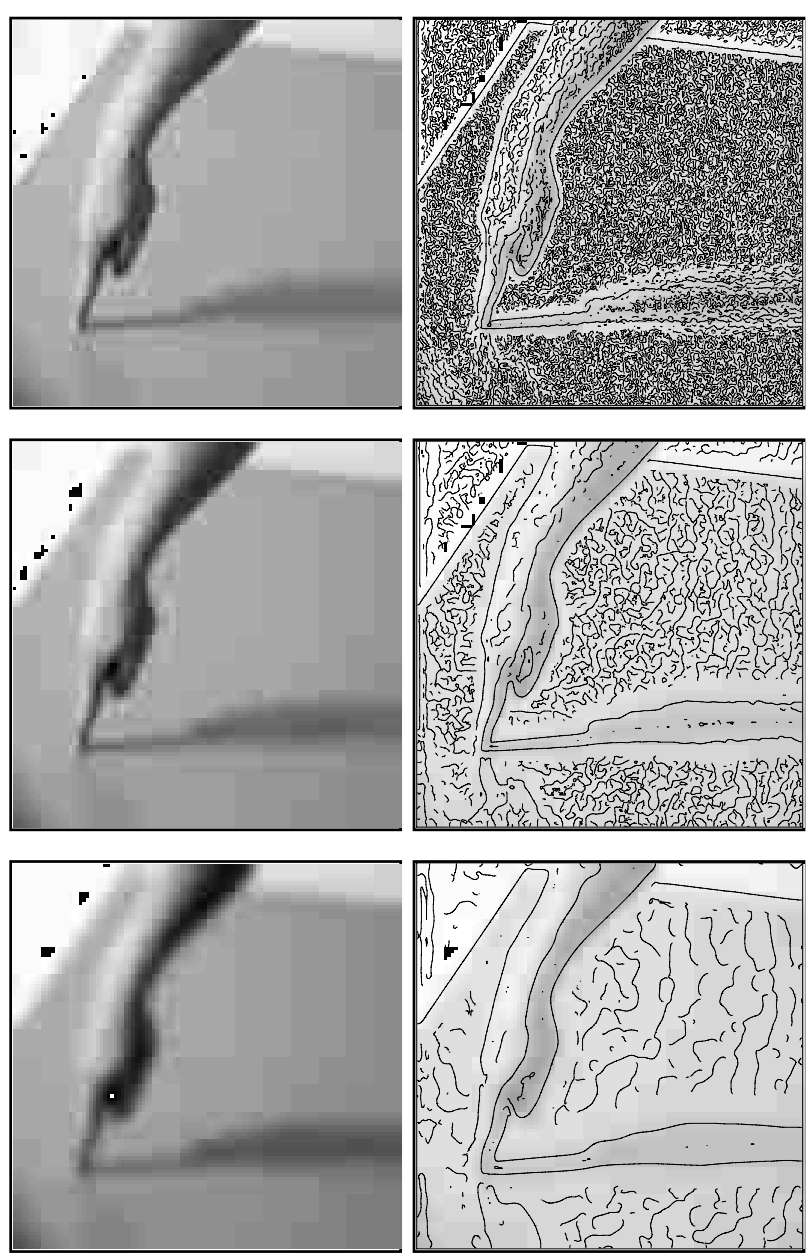

Figure 1: Edges computed at different scales in scale-space $(t=1.0,4.0$ and 16.0 from top to bottom) using a differential geometric formulation of non-maximum suppression. (Image size: $256^{*} 256$ pixels.)

smoothing will be substantial. Hence, to extract this edge with a reasonable trade-off between detection and localization properties, the only reasonable choice is to allow the scale levels to vary along the edge.

For this reason, and in view of the fact that the choice of scale levels crucially affects the performance of any edge detector, and different scale levels will, in general, be required in different parts of the image, we argue that it is essential to complement edge detectors by explicit mechanisms which automatically adapts the scale levels to the local image structure.

\section{Principle for scale selection}

The scale-space representation $L$ of a given signal $f$ is obtained by convolving $f$ by Gaussian kernels $g$ of various widths $t=\sigma^{2}$ (Witkin 1983, Koenderink 1984, Florack et al. 1992, Lindeberg 1994). From this representation, a scale-space derivative is defined by

$$
L_{x^{\alpha} y^{\beta}}(\cdot ; t)=\partial_{x^{\alpha} y^{\beta}}(g(\cdot ; t) * f)=g_{x^{\alpha} y^{\beta}}(\cdot ; t) * f .
$$

The output from these operators can be used as a basis for expressing a large number of visual operations, such as feature detection, matching, and computation of shape cues. A particularly convenient framework for expressing these is in terms of multi-scale differential invariants or singularities of these.

A basic problem for any such feature detector, however, concerns at what scales the image features should be extracted, and what image features should be regarded as significant. Early work addressing this problem was presented in (Lindeberg 1993a) for blob-like image structures. Then, in (Lindeberg 1993b, 1994) an extension was presented to other aspects of image structure. A general heuristic principle was proposed stating that local maxima over scales of (possibly nonlinear) combinations of normalized derivatives,

$$
\partial_{\xi}=\sqrt{t} \partial_{x},
$$

serve as indicators reflecting the spatial extent of corresponding image structures. Specifically, it was suggested that this idea could be used as a guide for scale selection algorithms, which automatically adapt the local scale of processing to the local image structure.

The subject of this article is to develop in more detail how this scale selection principle applies to the detection of one-dimensional image features, such as edges and ridges. For reasons that will become apparent later, we shall also extend this notion to scale selection based on $\gamma$-parameterized normalized derivatives

$$
\partial_{x, \gamma-\text { norm }}=t^{\gamma / 2} \partial_{x} .
$$

\section{Scale selection for edge detection}

At any image point, introduce a local coordinate system $(u, v)$ with the $v$-axis parallel to the gradient direction at that point, and the $u$-direction is perpendicular. Then, at any scale in scale-space, an edge point can be defined as a point at which the second directional derivative $L_{v v}$ in the $v$-direction is zero, and the third directional derivative $L_{v v v}$ is negative:

$$
\left\{\begin{array}{l}
L_{v v}=0 \\
L_{v v v}<0
\end{array}\right.
$$

If this definition is applied at all scales in scale-space, it sweeps out an edge surface. In view of the scale selection principle reviewed in the previous section, a natural extension of non-maximum suppression is by defining a scale-space edge as a curve on this surface, such 
that some suitably selected measure of edge strength $\mathcal{E}_{\gamma-\text { norm }} L$ assumes locally maxima with respect to scale on this curve. In differential geometric terms, a scalespace edge is thus defined as a connected set of points $\left\{(x, y ; t) \in \mathbb{R}^{2} \times \mathbb{R}_{+}\right\}$(a curve $\Gamma$ ) that satisfies

$$
\left\{\begin{array}{l}
\partial_{t}\left(\mathcal{E}_{\gamma-\text { norm }} L(x, y ; t)\right)=0 \\
\partial_{t t}\left(\mathcal{E}_{\gamma-\text { norm }} L(x, y ; t)\right)<0 \\
L_{\text {vv }}(x, y ; t)=0 \\
L_{\text {ขvข }}(x, y ; t)<0
\end{array}\right.
$$

Of course, there are several ways of expressing the condition that $\mathcal{E}_{\gamma-\text { norm }} L$ should assume local maxima over scales on the edge curve. In (4), this condition is formulated as in terms of the partial derivatives of $\mathcal{E}_{\gamma-\text { norm }} L$ with respect to the scale parameter. A natural alternative is to consider a directional derivative in the tangent plane to the edge surface, and to choose this direction as the steepest ascent direction of the scale parameter.

What remains to turn this idea into an operationally useful definition is to define the measure of edge strength. Based on the $\gamma$-parameterized normalized derivative concept in (2), we shall here consider the following two differential expressions:

$$
\begin{aligned}
\mathcal{G}_{\gamma-\text { norm }} L=t^{\gamma}\left(L_{x}^{2}+L_{y}^{2}\right), & \begin{aligned}
\mathcal{T}_{\gamma-\text { norm }} L=-t^{3 \gamma} & \left(L_{x}^{3} L_{x^{3}}+3 L_{x}^{2} L_{y} L_{x^{2} y}\right. \\
& \left.+3 L_{x} L_{y}^{2} L_{x y^{2}}+L_{y}^{3} L_{y^{3}}\right) .
\end{aligned}
\end{aligned}
$$

The first entity, the square gradient magnitude, is the presumably simplest measure of edge strength to think of. The second entity originates from the sign condition in the edge definition. These entities are both useful in practice, but have slightly different properties.

Qualitative properties. For a diffuse step edge, defined as the primitive function of a one-dimensional Gaussian, $f_{t_{0}}(x, y)=\int_{x^{\prime}=-\infty}^{x} g\left(x^{\prime} ; t_{0}\right) d x^{\prime}$, each of these measures of edge strength assume a unique maximum over scales at $t_{\mathcal{G}_{\gamma-\text { norm }}}=t_{\mathcal{I}_{\gamma-\text { norm }}}=\frac{\gamma}{1-\gamma} t_{0}($ for $x=0)$. Requiring this maximum to occur at $t_{0}$ gives $\gamma=\frac{1}{2}$.

For a Gaussian blob with $L(x, y ; t)=g\left(x, y ; t_{0}+t\right)$ with edges at $x^{2}+y^{2}=t_{0}+t$, the selected scales are $t_{\mathcal{G}_{\gamma-\text { norm }}}=\frac{\gamma}{3-\gamma} t_{0}$ and $t_{\mathcal{T}_{\gamma-\text { norm }}}=\frac{6 \gamma}{13-6 \gamma} t_{0}$.

Finally, for a local model of an edge bifurcation, expressed as $L(x ; t)=\frac{1}{4} x^{4}+\frac{3}{2} x^{2}\left(t-t_{b}\right)+\frac{3}{4}\left(t-t_{b}\right)^{2}$, with edges at $x_{1}(t)=\left(t_{b}-t\right)^{1 / 2}$ when $t \leq t_{b}$, we have $\left(\mathcal{G}_{\gamma-\text { norm }} L\right)\left(x_{1}(t) ; t\right)=4 t^{\gamma}\left(t_{b}-t\right)^{3}$, and the selected scales are $t_{\mathcal{G}_{\gamma-\text { norm }}}=\frac{\gamma}{3+\gamma} t_{b}$ and $t_{\mathcal{T}_{\gamma-\text { nor } m}}=\frac{3 \gamma}{5+3 \gamma} t_{b}$.

In summary, this short investigation shows that the scale selection method has the qualitative property of reflecting the degree of diffuseness of the edge. Moreover, since the edge strength decreases rapidly at a bi- furcation, this prevents the selected scale from being too close to the bifurcation scale.
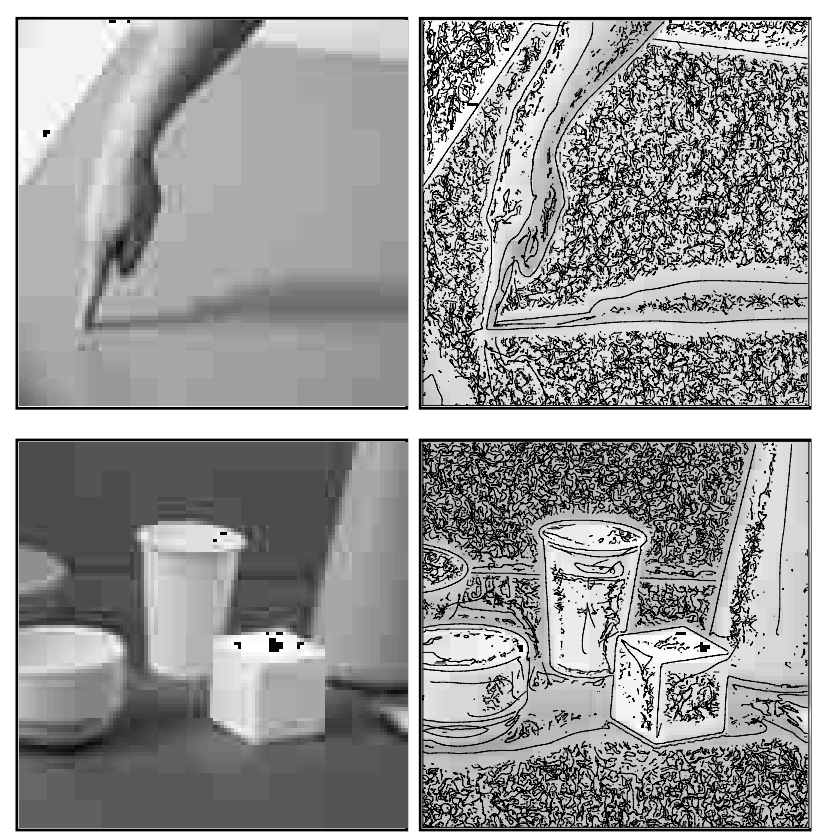

Figure 2: Results of edge detection with automatic scale selection based on local maxima over scales of $\mathcal{G}_{\gamma-\text { norm }} L$ (with $\gamma=\frac{1}{2}$ ). Image size: $256 \times 256$ pixels.

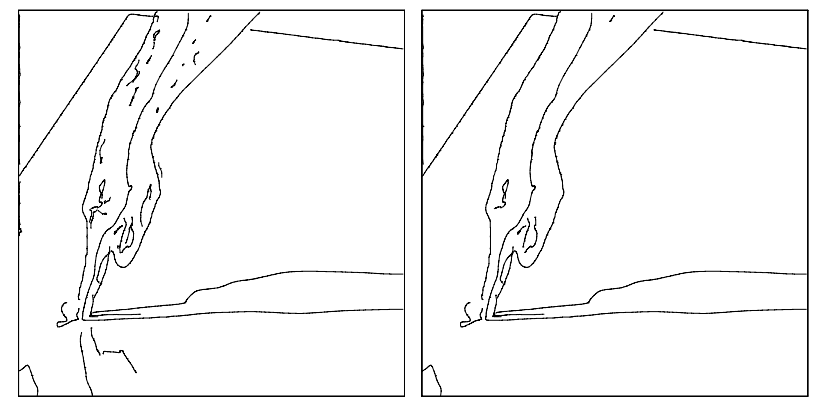

Figure 3: The 50 and 10 most significant edges from the arm image as ranked on the integrated $\gamma$-normalized gradient magnitude along the scale-space edge.

\section{Experiments: Edge detection}

Let us now apply the integrated edge detection scheme to different real-world images. In brief, edges are extracted as follows (Lindeberg 1996): The differential descriptors in the edge definition (4) (rewritten in terms of partial derivatives in Cartesian coordinates) are computed at a number of scales in scale-space. Then, a polygon approximation is constructed of the in- 


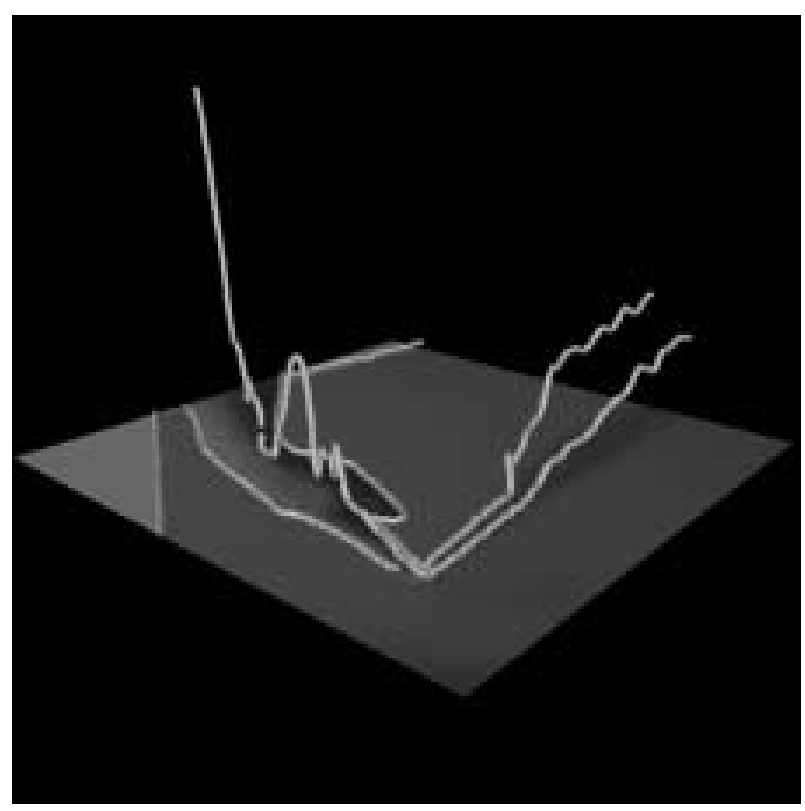

Figure 4: Three-dimensional view of the 10 most significant scale-space edges from the arm image drawn as curves in three-dimensional scale-space with the selected scale represented as the height over the image plane.

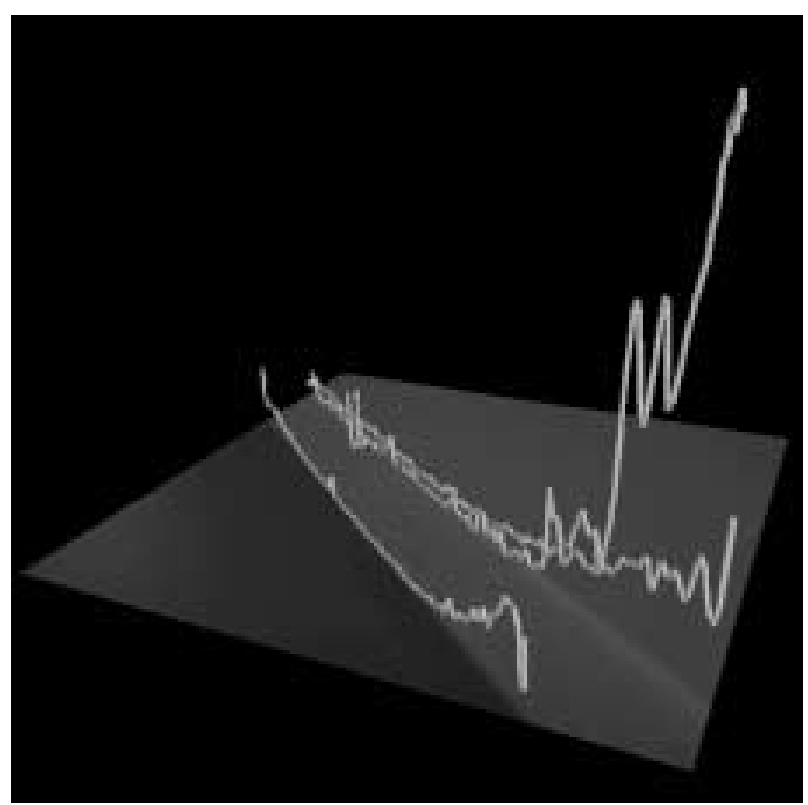

Figure 5: Three-dimensional view of the three strongest scale-space edges extracted from a detail of a table in which the edges are subject to out-of-focus blur. tersections of the two zero-crossing surfaces of $L_{v v}$ and $\partial_{t}\left(\mathcal{E}_{\gamma-\text { norm }}\right)$ that satisfy the sign conditions $L_{\text {vvv }}<0$ and $\partial_{t}\left(\mathcal{E}_{\gamma-\text { norm }}\right)<0$. Finally, a significance measure is computed for each edge by integrating the normalized edge strength measure along the curve

$$
\begin{aligned}
& G(\Gamma)=\int_{(x ; t) \in \Gamma} \sqrt{\left(\mathcal{G}_{\gamma-\text { norm }} L\right)(x ; t)} d s, \\
& T(\Gamma)=\int_{(x ; t) \in \Gamma} \sqrt[4]{\left(\mathcal{T}_{\gamma-\text { norm }} L\right)(x ; t)} d s .
\end{aligned}
$$

Fig. 2 shows the result of applying this scheme to two real-world images. As can be seen, the sharp edges due to object boundaries are extracted as well as the diffuse edges due to illumination effects (the occlusion shadows on the arm and the cylinder, the cast shadow on the table, as well as the reflection on the table). (Recall from fig. 1 that for this image it is impossible to capture the entire shadow edge at one scale without introducing severe shape distortions at the finger tip.)

Fig. 3 illustrates the ranking on significance obtained from the integrated edge strength along the curve. Whereas there are inherent limitations in using such an entity as the only measure of saliency, note that this measure captures essential information.

Fig. 4 gives a three-dimensional illustration of how the selected scale levels vary along the edges. The scale-space edges have been drawn as three-dimensional curves in scale-space, overlayed on a low-contrast copy of the original grey-level image in such a way that the height over the image plane represents the selected scale. Observe that coarse scales are selected for the diffuse edge structures due to illumination effects and that finer scales are selected for the sharp edge structures due to object boundaries. Fig. 5 shows another example, from a detail of table, for which the effects of focus blur are strong. Note how the selected scales capture the amount of out-of-focus blur along the edges.

Fig. 6 shows the result of applying edge detection with scale selection based on local maxima over scales of $\mathcal{T}_{\gamma-\text { norm }} L$ to an image containing a large amount of fine-scale information. From a first view, these results may appear very similar to the result of traditional edge detection at a fixed (very fine) scale. A more detailed study, however, reveals that a number of shadow edges are extracted, which would be impossible to detect at the same scale as the dominant fine-scale information. In this context, it should also be noted that the finescale edge detection in this case is not the result of any manual setting of tuning parameters. It is a direct consequence of the scale-space edge concept, and is the result of applying the same mechanism as extracts coarse scale levels for diffuse image structures. 

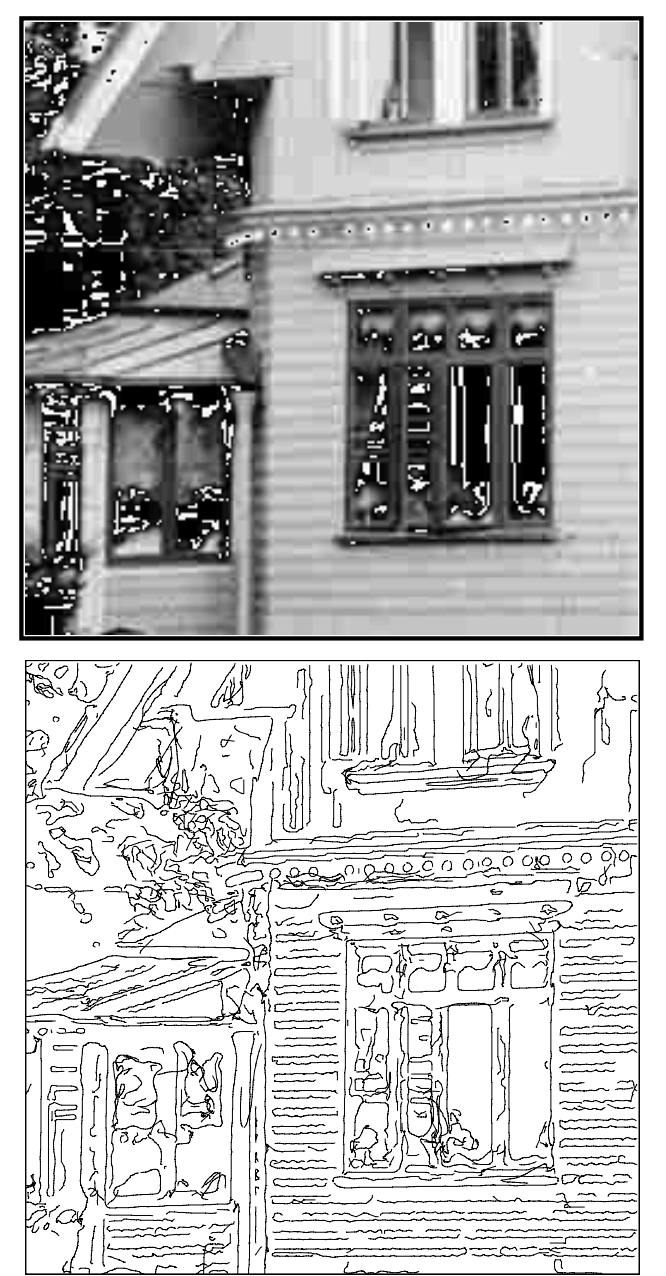

Figure 6: The 1000 strongest scale-space edges extracted using scale selection based on local maxima over scales of $\mathcal{T}_{\gamma-\text { norm }} L$ (with $\gamma=\frac{1}{2}$ ). (Image size: $256 \times 256$ pixels.)

\section{Scale selection for ridge detection}

By just slight modification, ridge detection algorithms can be formulated in a similar way. If we follow a differential geometric approach, and define a bright (dark) ridge point as a point for which the brightness assumes a maximum (minimum) in the main principal curvature direction (Haralick 1983; Eberly et al. 1994; Koenderink and van Doorn 1994), then in a local $(p, q)$ system characterized by the mixed second-order derivative being zero, this definition can be written

$$
\left\{\begin{array} { r l } 
{ L _ { p } } & { = 0 , } \\
{ L _ { p p } } & { < 0 , } \\
{ | L _ { p p } | } & { \geq | L _ { q q } | , }
\end{array} \quad \text { or } \quad \left\{\begin{array}{rl}
L_{q} & =0, \\
L_{q q} & <0, \\
\left|L_{q q}\right| & \geq\left|L_{p p}\right| .
\end{array}\right.\right.
$$

In analogy with section 3 , let us first sweep out a ridge surface in scale-space by applying this ridge definition at all scales. Then, given a measure $\mathcal{R}_{\gamma-\text { norm }} L$ of normalized ridge strength, define a scale-space ridge as a curve on this surface along which the ridge strength measure assumes local maxima with respect to scale. Here, we have considered the following measures:

$$
\begin{aligned}
\mathcal{M}_{\gamma-\text { norm }} L & =t^{\gamma} \max \left(\left|L_{p p}\right|,\left|L_{q q}\right|\right), \\
\mathcal{N}_{\gamma-\text { norm }} L & =t^{2 \gamma}\left(L_{p p}^{2}-L_{q q}^{2}\right)^{2} \\
\mathcal{A}_{\gamma-\text { norm }} L & =t^{2 \gamma}\left(L_{p p}-L_{q q}\right)^{2}
\end{aligned}
$$

For a Gaussian ridge defined by $f(x, y)=g\left(x ; t_{0}\right)$, it can be shown that for all these ridge strength measures the selected scale will then be $t_{\mathcal{R}_{\gamma-\text { norm }}}=\frac{2 \gamma}{3-2 \gamma} t_{0}$. Requiring this scale to be $t_{\mathcal{M}_{\gamma-\text { norm }}}=t_{0}$, lp gives $\gamma=\frac{3}{4}$.
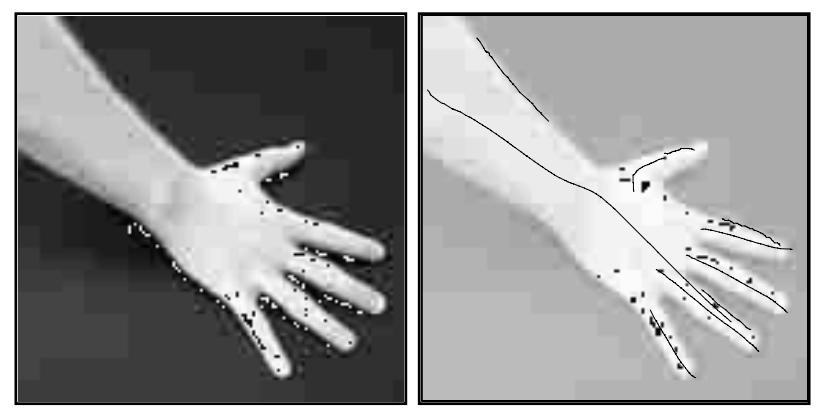

Figure 7: The 10 strongest bright ridges extacted using scale selection based on local maxima over scales of $\mathcal{A}_{\gamma-\text { norm }}$ (with $\gamma=\frac{3}{4}$ ). (Image size: $140 \times 140$.)

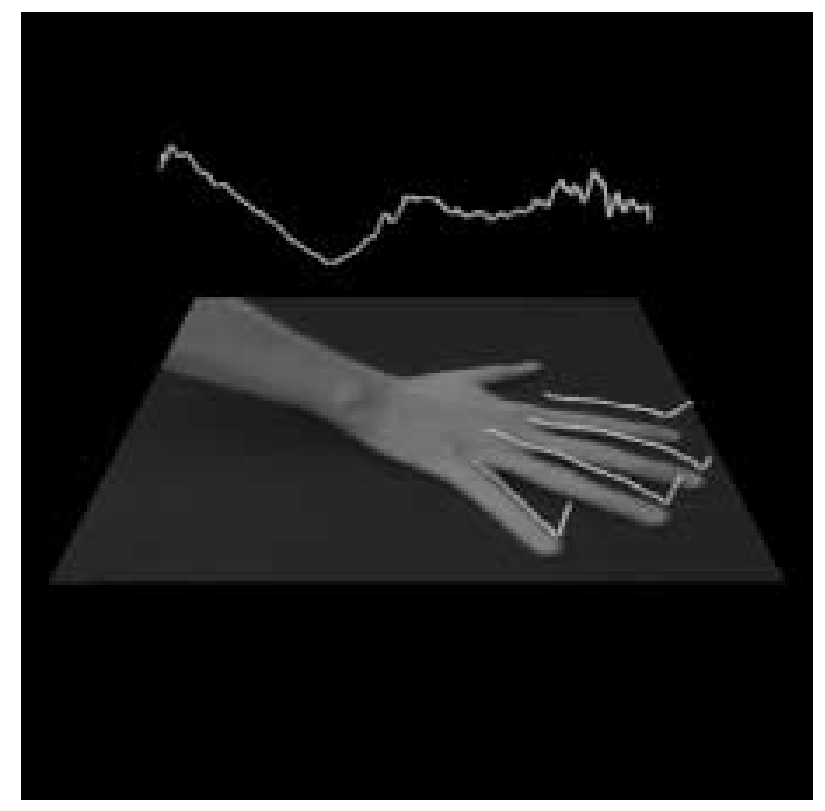

Figure 8: Three-dimensional view of the five strongest scale-space ridges extracted from the image in fig. 7 . 
Fig. 7 shows the result of applying such a ridge detector to an image and selecting the 10 strongest bright ridges, by integrating a measure of normalized ridge strength along the curve. Fig. 8 shows a threedimensional illustration of the scale-space ridges so obtained. Observe how a coarse-scale descriptor is extracted for the arm as a whole and that the individual fingers give rise to ridge curves at finer scales.

\section{Summary and discussion}

We have presented a framework for automatic local control of scale levels for edge detection and ridge detection. Compared to a traditional approach of defining and extracting such features from a grey-level image at a fixed level of scale (or a set of such fixed scales), we have proposed that it is more natural to define the concepts of edges and ridges as one-dimensional curves in the three-dimensional scale-space representation of the image (spanned by the spatial and scale dimensions).

A basic reason why such a definition is more useful is that in general situations a vision system cannot expect to know in advance what scales are appropriate for analysing the image structures in a given image. For these reasons we argue that a mechanism for automatic scale selection is a necessary complement to traditional multi-scale processing in general, and feature detection in particular. Specifically, concerning the edge detection problem, the resulting approach extends the notion of non-maximum suppression to a scale-space representation, and unifies this concept with several of the aims of diffuseness estimation schemes such as (Zhang and Bergholm 1993; Mallat and Zhong 1992). Concerning the ridge detection problem, the same type of framework captures several of the ideas behind the works in (Pizer et al. 1994; Eberly et al. 1994; Koller et al. 1995), and is based on local operations only.

Because of lack of space, this presentation is heavily condensed. A more extensive description can be found in (Lindeberg 1996), including further theory, motivations, algorithmic descriptions, as well as more detailed discussions about the relations to previous work.

Conclusion: Principles for scale selection. An essential aspect of this work (combined with the results in (Lindeberg 1993b, 1994)) is that it shows that detection of local maxima over scales of normalized differential invariants provides a consistent framework for automatic scale selection for detecting image features such as blobs, corners, edges and ridges.

Concerning the problem of computing local image deformations, such as optic flow and stereo matching, a related methodology for automatic scale selection is presented in (Lindeberg 1995). There, hypotheses about appropriate scales are generated from the scales at which normalized error measures assume local minima over scales.

These suggested principles may be far more general than the actual implementations presented so far.

\section{References}

F. Bergholm. Edge focusing. IEEE-PAMI, 9(6):726-741, 1987.

J. Canny. A computational approach to edge detection. IEEEPAMI, 8(6):679-698, 1986.

R. Deriche. Using Canny's criteria to derive a recursively implemented optimal edge detector. IJCV , 1:167-187, 1987.

D. Eberly, R. Gardner, B. Morse, S. Pizer, and C. Scharlach. Ridges for image analysis. JMIV , 4(4):353-373, 1994.

L. M. J. Florack, B. M. ter Haar Romeny, J. J. Koenderink, and M. A. Viergever. Scale and the differential structure of images. IVC, 10(6):376-388, 1992.

R. M. Haralick. Ridges and valleys in digital images. CVGIP, $22: 28-38,1983$.

R. M. Haralick. Digital step edges from zero-crossings of second directional derivatives. IEEE-PAMI, 6, 1984.

J. J. Koenderink and A. J. van Doorn. Two-plus-one-dimensional differential geometry. PRL, 15(5):439-444, 1994.

J. J. Koenderink. The structure of images. Biol. Cyb., 50:363$370,1984$.

T. M. Koller, G. Gerig, G. Szèkely, and D. Dettwiler. Multiscale detection of curvilinear structures in 2-D and 3-D image data. 5th ICCV, 864-869, Cambridge, MA, June 1995.

A. F. Korn. Toward a symbolic representation of intensity changes in images. IEEE-PA MI, 10(5):610-625, 1988.

T. Lindeberg. Detecting salient blob-like image structures and their scales with a scale-space primal sketch: A method for focus-of-attention. IJCV , 11(3):283-318, 1993.

T. Lindeberg. On scale selection for differential operators. 8th SCIA, 857-866, 1993.

T. Lindeberg. Scale-Space Theory in Computer Vision. Kluwer, Netherlands, 1994.

T. Lindeberg. Direct estimation of image deformations using visual front-end operators with automatic scale selection. $5 t h$ ICC V , 134-141, 1995.

$\mathrm{T}$. Lindeberg. Edge detection and ridge detection with automatic scale selection. ISRN KTH/NA/P-96/06-SE, 1996.

S. G. Mallat and S. Zhong. Characterization of signals from multi-scale edges. IEEE-PAMI, 14(7):710-723, 1992.

D. Marr and E. Hildreth. Theory of edge detection. Proc. Royal Soc. London, 207:187-217, 1980.

S. M. Pizer, C. A. Burbeck, J. M. Coggins, D. S. Fritsch, and B. S. Morse. Object shape before boundary shape: Scalespace medial axis. J. Math. Im. Vis., 4:303-313, 1994.

L. G. Roberts. Machine perception of three-dimensional solids. In J. T. Tippet et al, editor, Optical and Electro-Optical Information Processing, 159-197. MIT Press, 1965.

V. Torre and T. A. Poggio. On edge detection. IEEE-PAMI, $8(2): 147-163,1980$.

A. P. Witkin. Scale-space filtering. 8th IJCAI, 1019-1022, 1983.

W. Zhang and F. Bergholm. An extension of Marr's signature based edge classification and other methods for determination of diffuseness and height of edges, as well as line width. 4th ICCV, 183-191, 1993. 